\title{
Relación entre variables del forófito y la riqueza de epífitas vasculares en los Pantanos de Centla, Tabasco, México
}

\section{Relationship between phorophyte variables and vascular epiphyte richness in the Pantanos de Centla, Tabasco, Mexico}

\author{
Derio Antonio Jiménez-López ${ }^{1,4}$ (D), René de Jesús Roblero-Velasco' (D), Nayely Martínez-Meléndezl (D), \\ Gilberto Ocampo ${ }^{2}$ (D) y José Alberto Gallardo-Cruz ${ }^{3}$ (1)
}

1 Universidad de Ciencias y Artes de Chiapas, Instituto de Ciencias Biológicas, Libramiento Norte Poniente No. 1150 Col. Lajas Maciel, Tuxtla Gutiérrez, Chiapas, México.

2 Universidad Autónoma de Aguascalientes, Centro de Ciencias Básicas, Departamento de Biología, Avenida Universidad 940, 20131 Aguascalientes, México.

3 Centro del Cambio Global y la Sustentabilidad del Sureste (CCGSS), Calle Centenario del Instituto Juárez 128, Col. Reforma, 86080 Villahermosa, Tabasco, México.

4 Autor para la correspondencia: derio.a@hotmail.com

Citar como:

Jiménez-López, D. A., R. de J. Roblero-Velasco, N. Martínez-Meléndez, G. Ocampo y J. A. Gallardo-Cruz. 2017. Relación entre variables del forófito y la riqueza de epíitas vasculares en los Pantanos de Centla, Tabasco, México. Acta Botanica Mexicana 121: 125-137. DOI: http://dx.doi. org/10.21829/abml21.2017.1179

Recibido: 4 de noviembre de 2016 Revisado: 17 de mayo de 2017.

Aceptado: 5 de junio de 2017.

DOI:

http://dx.doi.org/10.21829/abml21.2017.1179

\section{RESUMEN:}

Antecedentes y Objetivos: Las epífitas vasculares son elementos poco comunes en manglares debido a que la salinidad del agua de mar impone condiciones extremas que dificultan su establecimiento. En el sur de México se han realizado estudios sobre las relaciones epífitas-forófito, principalmente en bosques montanos, pero hay pocos trabajos enfocados en los manglares. El objetivo de este trabajo fue analizar las relaciones entre la riqueza de epífitas y las características de los forófitos de Rhizophora mangle, especie amenazada de acuerdo a la NOM-059-SEMARNAT-2010, en la Reserva de la Biosfera "Pantanos de Centla", ubicada en el noreste del estado de Tabasco.

Métodos: Se evaluó la relación entre la riqueza de epífitas y algunas variables de los forófitos, como la altura, el diámetro a la altura del pecho (DAP) y la cobertura de raíces aéreas, mediante modelos de regresión lineal. Además, los forófitos se dividieron en cinco zonas verticales para analizar la distribución vertical de las epífitas aplicando los índices de Sørensen y Margalef.

Resultados clave: Se registraron 17 especies de epífitas. Orchidaceae y Bromeliaceae fueron las familias con mayor riqueza (seis especies cada una). El género más representativo fue Tillandsia (Bromeliaceae) con cinco especies. La riqueza de epífitas estuvo significativamente relacionada con la cobertura de raíces aéreas y el DAP de los forófitos. Las zonas I y III de los forófitos fueron las más similares ya que compartieron $47 \%$ del total de las especies registradas. La zona I (raíces aéreas) fue la más rica en especies de epífitas. En la zona V (parte exterior de la copa) solo se encontró a Selenicereus testudo (Cactaceae), especie también hallada en el resto de las zonas. El área de manglar estudiado presenta una riqueza relativamente alta de epífitas pese a la alta salinidad presente en su hábitat inundado. Conclusiones: La variación de los tamaños de los forófitos determinó la riqueza de epífitas. Las raíces aéreas de $R$. mangle son un nicho adicional para la colonización de epífitas y no solamente sirven como soporte al suelo inestable del ecosistema de manglar.

Palabras clave: Bromeliaceae, composición florística, distribución vertical, manglares, Orchidaceae, Rhizophora mangle.

\section{ABSTRACT:}

Background and Aims: Vascular epiphytes are rare elements of mangrove-dominated forests because sea salinity causes extreme conditions that difficult their establishment. In southern Mexico, most of the studies about the relationships between epiphytes and phorophytes have been carried out in montane forests, while the mangrove forests have received little attention. Our aim was to analyze the relationships between epiphytic species richness and the phorophyte characteristics of Rhizophora mangle, an endangered species under the NOM-059-SEMARNAT- 2010, in the "Pantanos de Centla" Biosphere Reserve located in the north-east of the state of Tabasco.

Methods: The relation between species richness of epiphytes and several variables of the phorophytes, such as the aerial root coverage, diameter at breast height $(\mathrm{DBH})$ and height, was evaluated using linear regression models. In addition, phorophytes were divided into five vertical zones to analyze the vertical distribution of the epiphytes using the Sørensen and Margalef indexes.

Key results: Seventeen species of epiphytes were recorded. Orchidaceae and Bromeliaceae were the families with the highest species richness (six each). The most representative genus was Tillandsia (Bromeliaceae) with five species. The epiphytic species richness was significantly correlated with phorophyte aerial root coverage and DBH. The phorophyte zones I and III were the most similar because they shared $47 \%$ of all recorded species. The zone I (aerial roots) showed the highest epiphyte species richness. In the zone V (outer portion of the canopy) only Selenicereus testudo (Cactaceae) was registered, a species that was found in all five zones of the phorophyte. The mangrove area studied has a relatively high epiphyte richness in spite of the high salinity present in its flooded habitat.

Conclusions: Epiphytic species richness was determined by the variation in size of the phorophytes. The aerial roots of $R$. mangle are an additional niche for the colonization of epiphytes and not just a supporting structure for the unstable soil of the mangrove ecosystem.

Key words: Bromeliaceae, floristic composition, mangroves, Orchidaceae, Rhizophora mangle, vertical distribution. 


\section{INTRODUCCIÓN}

Los manglares son extensas áreas de bosques que bordean las zonas intermareales costeras, se localizan en las zonas tropicales y subtropicales del planeta (Spalding et al., 2010; Giri et al., 2011). Las especies arbóreas dentro de este ecosistema presentan características y especializaciones morfológicas y funcionales que les permiten desarrollarse ante condiciones difíciles en un medio acuático salino de suelos inestables; por ejemplo, órganos especiales de respiración y sostén, y metabolismo adaptado a altas concentraciones de sal y largo poder germinativo (Tomlinson, 2016). Una notable adaptación de uno de los géneros con más diversidad en los manglares de México, Rhizophora L., son las raíces aéreas (Rodríguez-Zúñiga et al., 2013). Esta adaptación fisiológica está directamente relacionada con la naturaleza anaeróbica de los sustratos en los pantanos, ya que el sistema de raíces permite que Rizophora realice el intercambio gaseoso en las condiciones anaerobias del suelo inundado de los pantanos (Poungparn y Komiyama, 2013; Tomlinson, 2016). La mayor parte de la biomasa neta de Rhizophora se da en estos sistemas de raíces aéreas, las cuales soportan biomecánicamente la copa del árbol y actúan como contrafuerte flotante (Tomlinson, 2016). Las condiciones impuestas por la salinidad y otras de origen mecánico (influencia de olas, mareas y corrientes) limitan la persistencia de otros gremios de plantas además de los árboles, como es el caso de las epífitas vasculares, las cuales son poco comunes en los manglares, debido a las características del árbol hospedero (tipo de corteza y arquitectura) o la exposición a la salinidad, entre otros factores (Gómez y Winkler, 1991; Zotz y Reuter, 2009; Rodríguez-Zúñiga et al., 2013). Sin embargo, otros ecosistemas de manglar pueden albergar una alta diversidad de epífitas vasculares, por ejemplo los manglares de Asia (Giesen et al., 2007).

Las epífitas, incluyendo orquídeas, aráceas, bromelias y helechos, entre otras, son plantas que crecen principalmente sobre árboles hospederos. Esta relación se considera mutualista, ya que las epífitas no tienen una relación fisiológica con sus forófitos (no son parásitas) y únicamente los utilizan para sujetarse del tronco y las ramas (Benzing, 1990; Zotz, 2013; Krömer et al., 2014). La ventaja de crecer sobre otras plantas es acceder a una cantidad mayor de luz solar. Sin embargo, esta forma de vida es desfavorable en lo que a captación de agua y nutrientes se refiere, por lo que las epífitas han desarrollado una serie de adaptaciones morfológicas, anatómicas y fisiológicas (Zotz, 2016). Entre éstas se encuentran modificaciones presentes en hojas, p. ej., mayor suculencia (las células del mesófilo son más numerosas y de mayor tamaño), el desarrollo de una cutícula gruesa, presencia de metabolismo CAM y mayor tamaño de escamas (en Bromeliaceae), que ayudan en la absorción del agua y nutrientes directamente hacia el interior de la hoja (Benzing, 1990; Gómez y Winkler, 1991; Zotz y Andrade, 2002; Reyes-García et al., 2012). La capacidad de las epífitas para colonizar a los hospederos depende de características como la arquitectura, el tamaño (que corresponde con la edad), el tipo de corteza y la variación microclimática, que determinan la estratificación vertical desde la parte alta del dosel hacia el interior del bosque (Benzing, 1990; Gradstein et al., 2003; Krömer et al. 2007a; Martínez-Meléndez et al., 2008). La estratificación vertical de epífitas en ecosistemas de manglar puede variar a diferentes alturas y zonas de un mismo hospedero, dependiendo de la altura del árbol y la cobertura alta del dosel, que amortiguan la gran variabilidad de las condiciones climáticas (Cach-Pérez et al., 2013).

El gran interés por definir qué variables estructurales del forófito influyen en la colonización y distribución de las epífitas en un manglar, ubicado en La Reserva de la Biosfera "Pantanos de Centla" en el noroeste del estado de Tabasco en México, llevó a plantearnos las siguientes preguntas: ¿Existe una relación directa entre la riqueza de especies de epífitas vasculares con la altura, el diámetro a la altura del pecho (DAP) y el área de la cobertura de las raíces aéreas en forófitos de Rhizophora mangle L.?, ¿En qué zona(s) vertical(es) del forófito se observará la mayor riqueza de epífitas vasculares?, ¿Las raíces aéreas de $R$. mangle contribuyen como una zona adicional para la colonización de epífitas vasculares? Nuestras hipótesis son las siguientes: (1) Las variables de los forófitos determinan una mayor riqueza de epífitas vasculares, es decir a mayor altura, DAP y área de la cobertura de raíces aé- 
reas de los forófitos, mayor riqueza de epífitas vasculares, (2) El área de la cobertura de raíces aéreas contendrá una riqueza similar al dosel en forófitos de $R$. mangle, y (3) Aunque no se tomaron datos del porcentaje de salinidad en el sitio de estudio, indirectamente suponemos que la hipersalinidad del agua de mar impone condiciones extremas para el establecimiento de epífitas en áreas de manglar, por lo que estas serán escasas en el área de estudio.

\section{Materiales y Métodos}

\section{Área de estudio}

El estudio se efectuó en la laguna El Cometa (Fig. 1), ubicada dentro de la Reserva de la Biosfera "Pantanos de Centla" (302,706 ha), al noreste del estado de Tabasco, México. La laguna se encuentra entre los $18^{\circ} 27^{\prime}$ y $18^{\circ} 28^{\prime}$ de latitud $\mathrm{N}$ y $92^{\circ} 27^{\prime}$ y $92^{\circ} 28^{\prime}$ de longitud W y abarca 67 ha. Está ubicada en la región hidrológica Grijalva - Usumacinta (INE, 2000; Rodríguez-Rodríguez, 2002). El tipo de vegetación dominante en este ecosistema es manglar, dominado por $R$. mangle, especie amenazada de acuerdo a la NOM-059-SEMARNAT-2010, que se mezcla con selva mediana subperennifolia de Bucida buceras L. o pukteal (Miranda y Hernández X., 1963). La precipitación media anual en la región es de 1693 $\mathrm{mm}$, y la mayoría cae durante los meses de junio a octubre. La temperatura media anual es de $27^{\circ} \mathrm{C}$ (Kauffman et al., 2016).

\section{Selección de los forófitos}

El estudio se realizó en el mes de julio del 2014. Debido a limitaciones técnicas y logísticas de acceso a sitios remotos

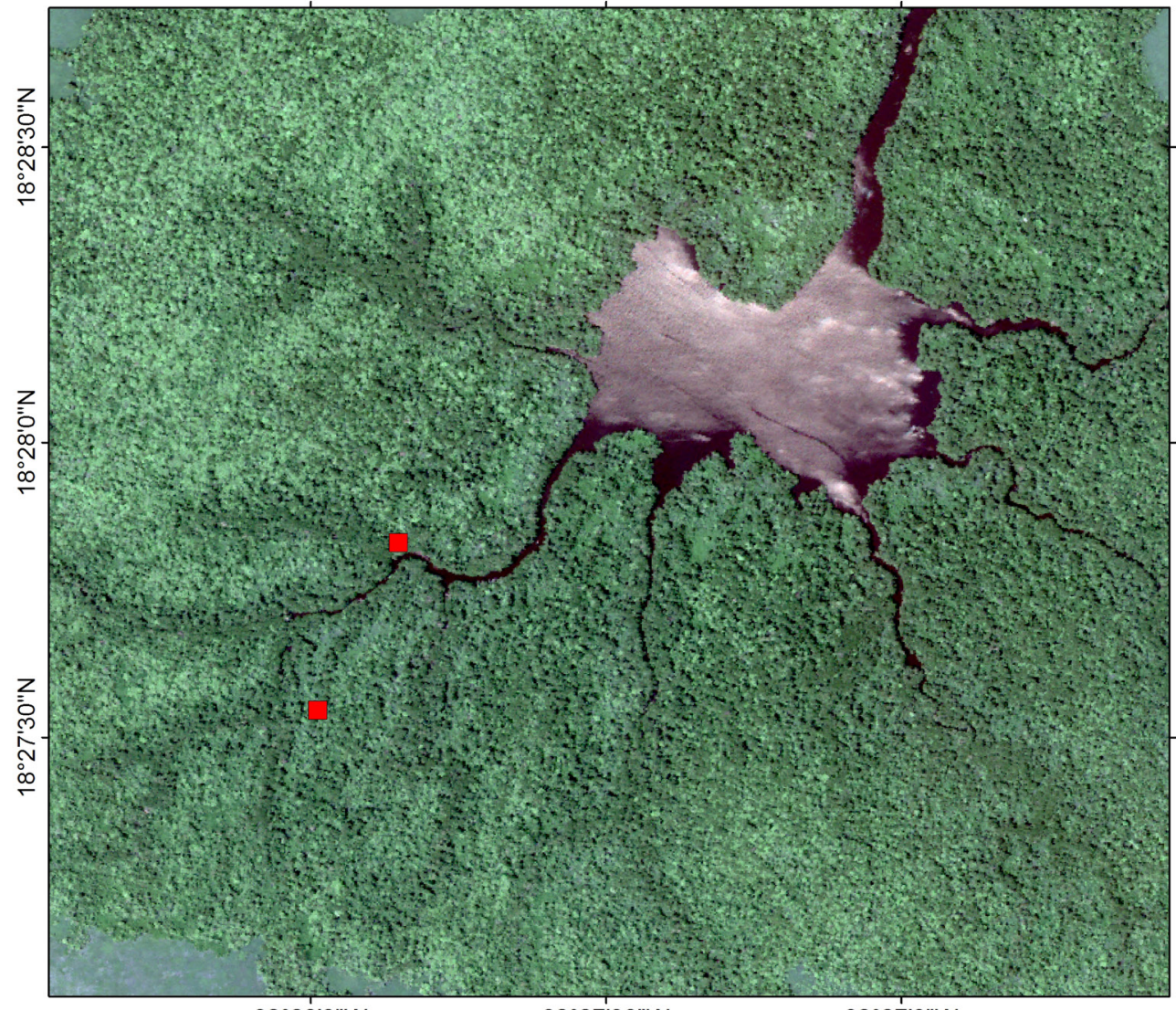

$92^{\circ} 28^{\prime} 0 " \mathrm{~W}$

$92^{\circ} 27^{\prime} 30^{\prime \prime} \mathrm{W}$

$92^{\circ} 27^{\prime} 0 " \mathrm{~W}$

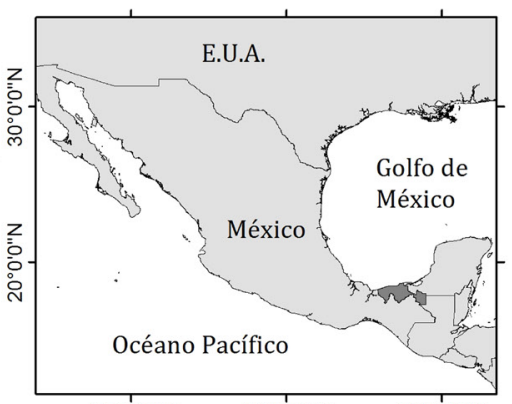

$110^{\circ} 0^{\prime} \mathrm{O}^{\prime \prime} \mathrm{W} \quad 100^{\circ} \mathrm{O}^{\prime} \mathrm{O} \quad \mathrm{W} \quad 90^{\circ} \mathrm{O}^{\prime} \mathrm{O} \mathrm{W} \mathrm{W}$
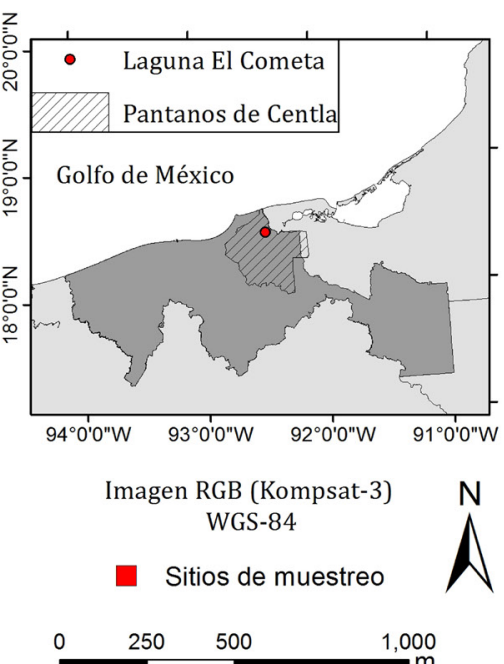

Figura 1: Ubicación del área de estudio y puntos de muestreo en la laguna El Cometa en la Reserva de la Biosfera "Pantanos de Centla", Tabasco, México. 
e inundables, se trazaron dos parcelas de $50 \times 50 \mathrm{~m}(0.5$ ha cada una) en sitios seleccionados al azar. En cada cuadro se censaron los forófitos de $R$. mangle con un diámetro mínimo a la altura del pecho (DAP) $\geq 10 \mathrm{~cm}$ (modificado de Cach-Pérez et al., 2013), para representar a la mayoría de los forófitos con posible colonización de epífitas, debido a que el promedio de altura de los forófitos muestreados es $>17 \mathrm{~m}$ y por debajo de este DAP la presencia de epífitas fue escasa. Además, se ha encontrado una relación directa entre el DAP de los forófitos y el incremento en la riqueza de especies epífitas (Flores-Palacios y García-Franco, 2008).

Se midieron las siguientes variables de los forófitos: 1) altura, tomada desde la parte baja del conjunto de raíces aéreas que están en contacto con el suelo y/o agua, hasta la parte alta del dosel, 2) diámetro a la altura del pecho (DAP), a $1.3 \mathrm{~m}$ por encima de la última raíz aérea que sostiene al forófito y tienen acceso al suelo y, 3) área de la cobertura de raíces aéreas $\left(\mathrm{m}^{2}\right)$. Se trazaron dos líneas perpendiculares dentro de un círculo donde se encontró la mayor concentración del conjunto de raíces para después obtener el área del círculo en $\mathrm{m}^{2}$. Debido a que en otros tipos de bosques tropicales los forófitos no presentan raíces aéreas, en $R$. mangle podrían ser importantes como una zona adicional para la colonización de epífitas vasculares.

\section{Censo de epífitas}

Se contabilizó la presencia-ausencia de las especies de epífitas vasculares presentes en cada forófito, que fue dividido en cinco zonas verticales (modificado de Johansson, 1974) para determinar la riqueza de epífitas en diferentes estratos: (I) raíces aéreas, (II) tronco, no se tomaron en cuenta aquellas raíces aéreas que contenían la zona del tronco y que no tocaban el suelo, por tal motivo tampoco eran parte de la zona de raíces aéreas, (III) base de la copa, (IV) parte media de la copa y, (V) parte exterior de la copa (Fig. 2).

La delimitación de plantas individuales suele ser difícil en las epífitas vasculares. Se definió a un individuo como un grupo de rizomas y hojas pertenecientes a una especie y que forma una posición delimitada, excluyendo las plántulas pequeñas que a menudo no se pueden identificar, y además pueden ser un componente inestable por

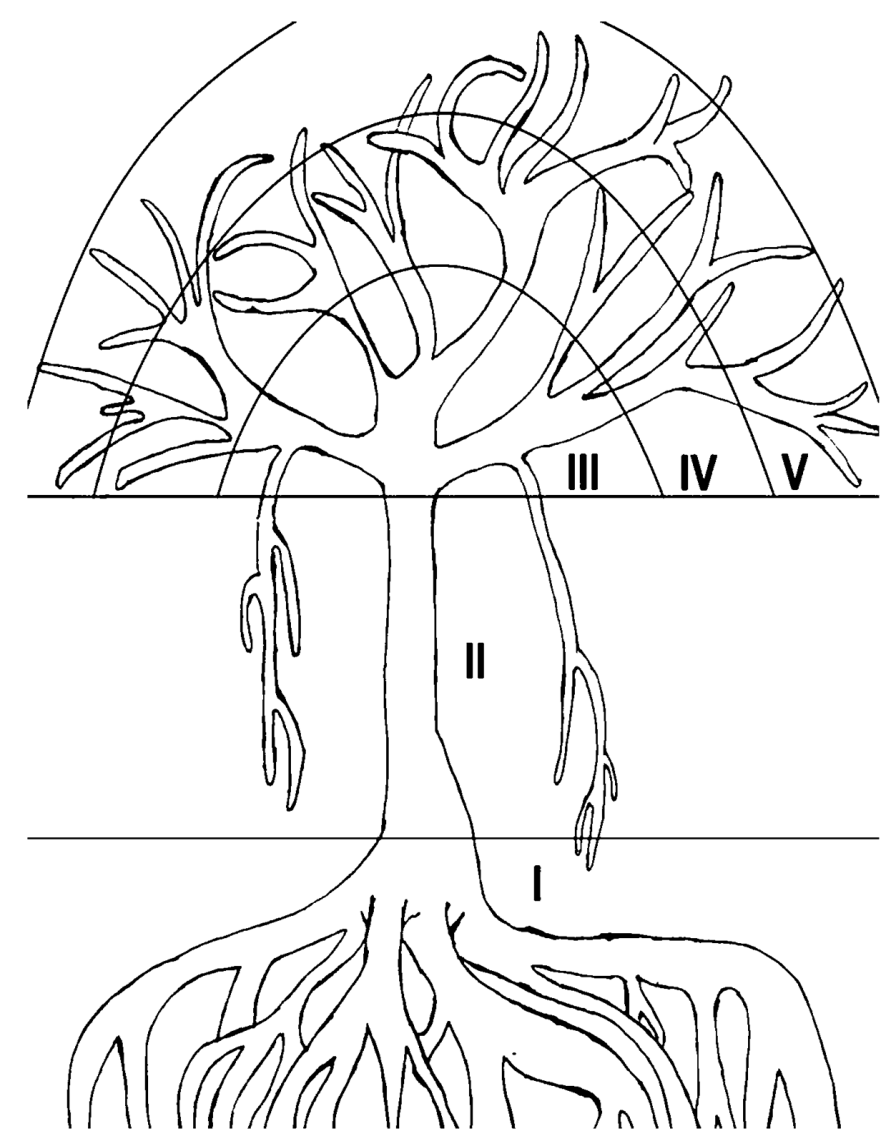

Figura 2: División del forófito considerada para este estudio. Estratificación vertical modificada de Johansson (1974). Ilustración: René Roblero Velasco.

las altas tasa de mortalidad en las partes altas del dosel (Sanford, 1968; Zotz y Schultz, 2008; Cach-Pérez et al., 2013). Para cada especie de epífita se colectaron especímenes para ser herborizados de acuerdo a Lot y Chiang (1986); los ejemplares obtenidos fueron depositados en el herbario HEM del Instituto de Ciencias Biológicas de la Universidad de Ciencias y Artes de Chiapas en Tuxtla Gutiérrez, Chiapas, México.

\section{Análisis de datos}

\section{Estructura y riqueza vertical}

Para saber si había similitud florística entre las zonas verticales del forófito, se efectuó un análisis de agrupamiento (UPGMA) con el índice de Sørensen, el cual permite com- 
parar dos o más zonas mediante la presencia/ausencia de especies en cada una de ellas (Moreno, 2001). Para determinar qué zona del forófito contenía la mayor riqueza de epífitas, se calculó el índice de Margalef, que estima la diversidad de una comunidad con base a la distribución numérica de los individuos de las diferentes especies en función del número de individuos existentes en la muestra analizada; en este caso, valores inferiores a dos indican baja diversidad y valores superiores a cinco son considerados como alta biodiversidad (Margalef, 1995). Para ambos casos se utilizó la plataforma estadística R (R Core Team, 2015).

\section{Relación entre el tamaño del forófito y la rique-} za de epífitas

Se ajustaron modelos de regresión lineal para explorar la relación entre las tres variables de los forófitos (altura, DAP y área de raíces aéreas) con la riqueza de epífitas con la plataforma estadística R (R Core Team, 2015). La altura y el DAP de los forófitos son las variables que más se utilizan en estudios de epífitas y pueden ser un tanto predecibles, es decir a mayor altura y DAP, mayor riqueza de epífitas vasculares (Flores-Palacios y García-Franco, 2006; Haro-Carrion et al., 2009; Wolf et al., 2009). Sin embargo, la tendencia en ecosistemas de manglar no podría ser tan predecible, puesto que el área de la cobertura de raíces aéreas es una variable que no se ha usado para estudios de epífitas en manglares.

\section{Resultados}

\section{Riqueza y composición florística}

Se registraron 17 especies de epífitas vasculares pertenecientes a cinco familias y 11 géneros (Cuadro 1). E1 82\%

Cuadro 1: Listado de especies de epífitas vasculares en Rhizophora mangle L. de la laguna El Cometa, Reserva de la Biosfera "Pantanos de Centla", indicando presencia $=1$ y ausencia $=0$ de las especies en las zonas verticales del forófito, modificado de Johansson (1974).

\begin{tabular}{|c|c|c|c|c|c|c|}
\hline \multirow[t]{2}{*}{ Familia } & \multirow[t]{2}{*}{ Especie } & \multicolumn{5}{|c|}{ Zonas verticales del forófito } \\
\hline & & I & II & III & IV & $\mathrm{V}$ \\
\hline Araceae & Anthurium schlechtendalii Kunth & 1 & 0 & 0 & 0 & 0 \\
\hline \multirow[t]{2}{*}{ Cactaceae } & Selenicereus testudo (Karw. ex Zucc.) Buxb. & 1 & 1 & 1 & 1 & 1 \\
\hline & Selenicereus sp. & 0 & 1 & 1 & 1 & 0 \\
\hline \multirow[t]{6}{*}{ Bromeliaceae } & Aechmea bracteata (Sw.) Griseb. & 1 & 0 & 1 & 0 & 0 \\
\hline & Tillandsia balbisiana Schult. f. & 1 & 0 & 1 & 0 & 0 \\
\hline & Tillandsia brachycaulos Schltdl. & 1 & 0 & 1 & 1 & 0 \\
\hline & Tillandsia bulbosa Hook. & 0 & 0 & 1 & 1 & 0 \\
\hline & Tillandsia streptophylla Scheidw. ex C. Morren & 0 & 0 & 1 & 1 & 0 \\
\hline & Tillandsia $\mathrm{sp}$. & 1 & 1 & 1 & 0 & 0 \\
\hline \multirow[t]{6}{*}{ Orchidaceae } & Catasetum integerrimum Hook. & 1 & 1 & 1 & 0 & 0 \\
\hline & Encyclia alata (Bateman) Schltr. & 1 & 0 & 0 & 0 & 0 \\
\hline & Myrmecophila aff. tibicinis (Bateman) Rolfe & 1 & 1 & 0 & 0 & 0 \\
\hline & Notylia barkeri Lindl. & 1 & 0 & 0 & 0 & 0 \\
\hline & Trichocentrum cosymbephorum (C. Morren) R. Jiménez \& Carnevali & 1 & 0 & 1 & 0 & 0 \\
\hline & Trichocentrum $\mathrm{sp.}$ & 1 & 0 & 0 & 0 & 0 \\
\hline \multirow[t]{2}{*}{ Polypodiaceae } & Microgramma nitida (J. Sm.) A.R. Sm. & 1 & 1 & 0 & 0 & 0 \\
\hline & Phlebodium decumanum (Willd.) J. Sm. & 1 & 0 & 1 & 0 & 0 \\
\hline
\end{tabular}


de las especies se encontraron en las raíces aéreas (Cuadro 1; Fig. 4). Las familias más representativas son Orchidaceae y Bromeliaceae, ambas con seis especies; siendo los géneros Tillandsia L. (Bromeliaceae) y Trichocentrum Poepp. \& Endl. (Orchidaceae), con cinco y dos especies respectivamente, los géneros con mayor representación.

\section{Estructura y distribución vertical}

Las zonas verticales del forófito más similares entre sí fueron I y III (IS=0.64) que comparten $47 \%$ del total de las especies (Fig. 3). Esto corresponde a ocho especies (Aechmea bracteata (Sw.) Griseb., Catasetum integerrimum Hook., Selenicereus testudo (Karw. ex Zucc.) Buxb., Phlebodium decumanum (Willd.) J. Sm., Tillandsia balbisiana Schult. f., T. brachycaulos Schltdl., Tillandsia sp. y Trichocentrum cosymbephorum (C. Morren) R. Jiménez \& Carnevali. Las zonas II y IV comparten dos especies ( $S$. testudo y Selenicereus sp.). En la zona V solo se halló a $S$. testudo, la cual también se encontró en todos los demás estratos del forófito. Según el cálculo de la riqueza obtenida mediante el índice de Margalef, la zona I es la que presenta mayor riqueza (5.17), seguida de III (4.17), II (2.79) y IV (2.48).
Relación entre el tamaño del forófito y la riqueza de epífitas

El modelo de regresión lineal entre la altura vs. riqueza, aunque fue significativa positiva, es la que presento menor inercia con la riqueza de epífitas $(\mathrm{r}=0.29, p=0.001)$ (Fig. $5 \mathrm{~A})$. El área de la cobertura de raíces aéreas y la riqueza

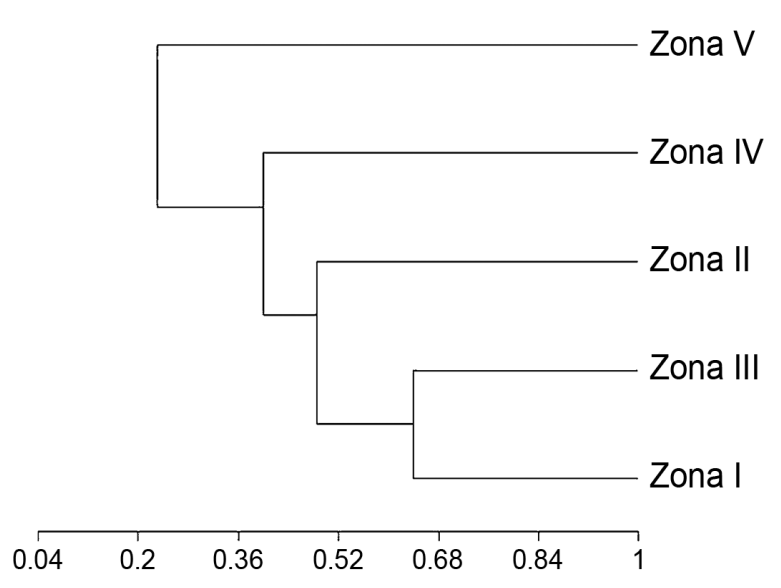

Figura 3: Análisis de agrupamiento (UPGMA) que muestra la relación de la similitud, utilizando el índice de similitud de Sørensen, entre los diferentes estratos verticales evaluados en forófitos de Rhizophora mangle $\mathrm{L}$.
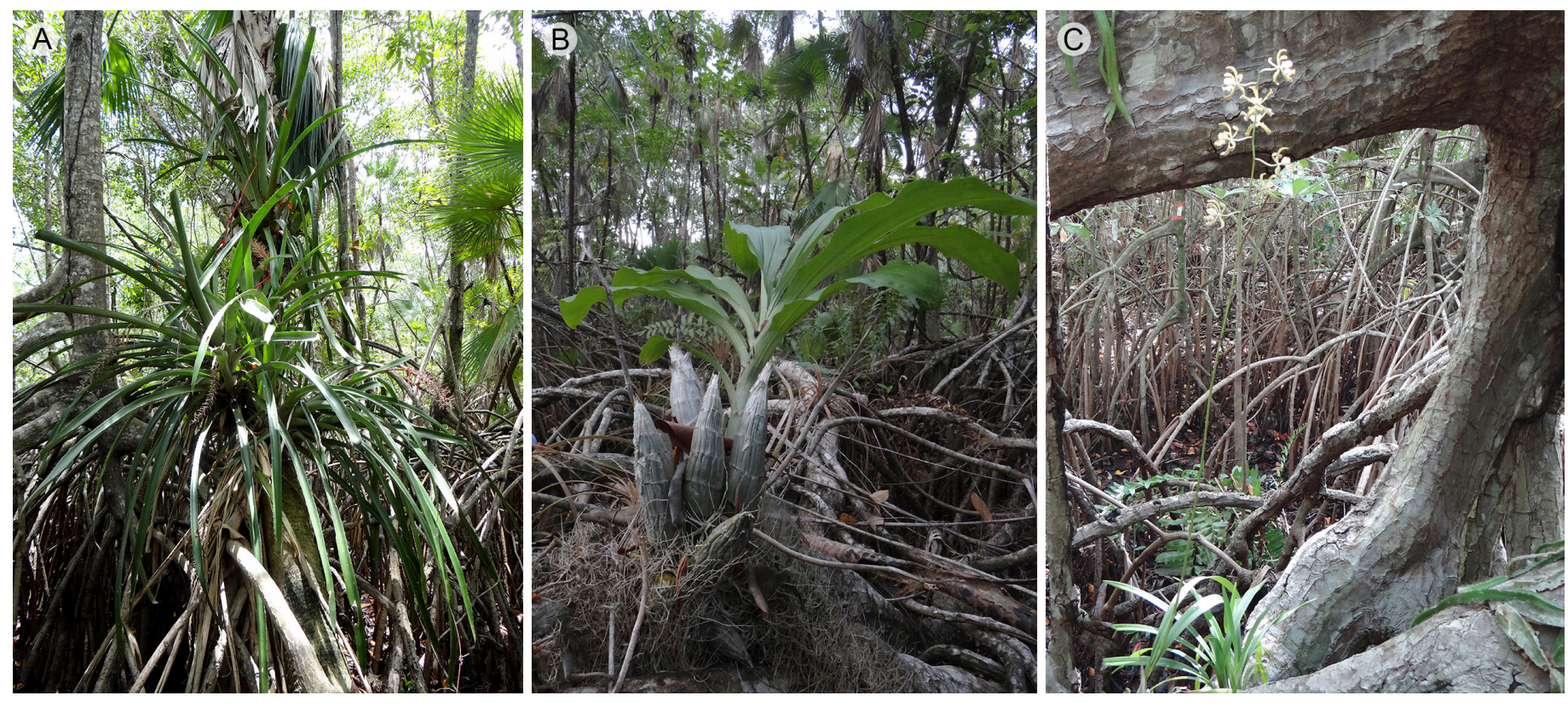

Figura 4. Epífitas en raíces aéreas de Rhizophora mangle L. A. Aechmea bracteata (Sw.) Griseb. (Bromeliaceae); B. Catasetum integerrimum Hook. (Orchidaceae); C. Encyclia alata (Bateman) Schltr. (Orchidaceae). 

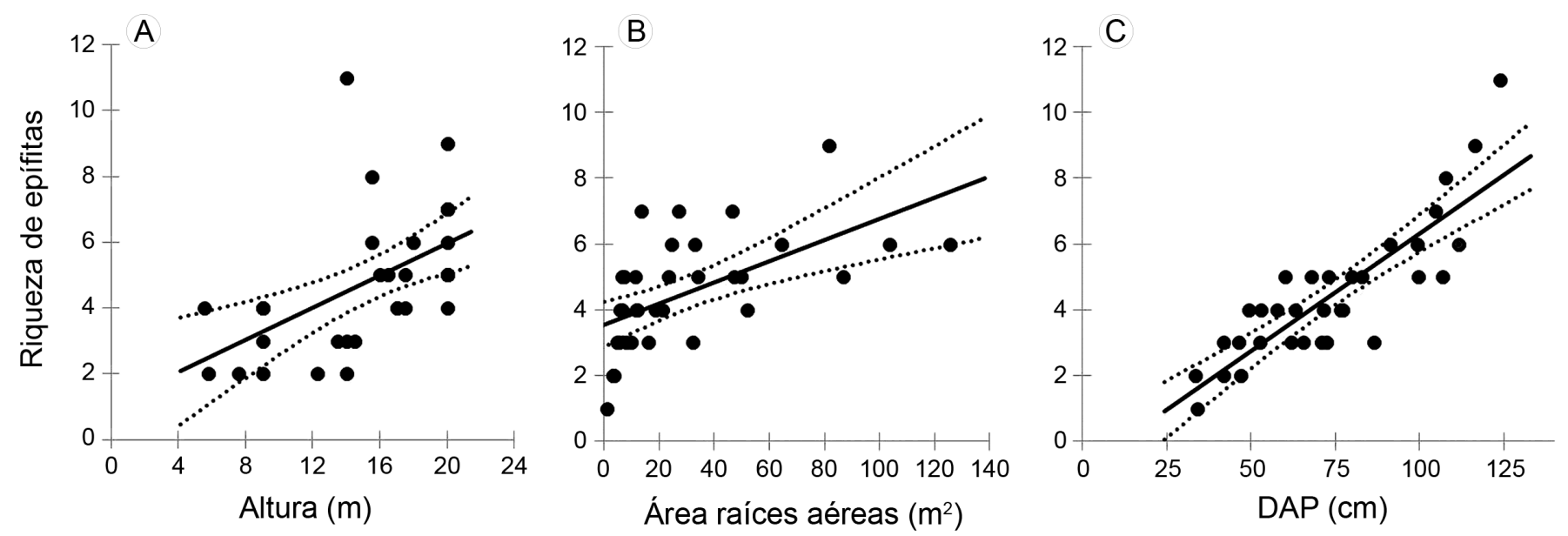

Figura 5: Relación entre la riqueza de especies y las variables del forófito. A. altura vs. riqueza $\left(\mathrm{a}=1.0732, \mathrm{~b}=3.2300, \mathrm{r}^{2}=0.29, p=0.001\right) ; \mathrm{B}$. área de raíces vs. riqueza de epífitas $\left(\mathrm{a}=3.5483, \mathrm{~b}=3.2300, \mathrm{r}^{2}=0.34, p<0.0001\right)$; C. DAP vs. riqueza $\left(\mathrm{a}=0.8158, \mathrm{~b}=7.1323, \mathrm{r}^{2}=0.72, p<0.0001\right)$.

de epífitas fue significativa positiva $(\mathrm{r}=0.34, p<0.0001)$ (Fig. 5B) y el DAP vs. riqueza fue la que presentó mayor relación $(\mathrm{r}=0.72, p<0.0001)$ (Fig. 5C).

\section{Discusión}

\section{Riqueza y composición florística}

Se encontraron 17 especies que corresponden a $85 \%$ de las epífitas vasculares reportadas para la Reserva de la Biósfera "Pantanos de Centla" (Guadarrama-Olivera y Ortiz-Gil, 2000). Esta riqueza es similar a la reportada para el manglar de Jicacal, Veracruz con 22 especies, pero diferente a las 43 especies de Sontecomapan en el mismo estado y las siete reportadas para los Manglares de Marismas Nacionales en Nayarit (Valdez-Hernández, 2000). De esta manera la tercera hipótesis se rechaza parcialmente, debido a que la riqueza de epífitas en este manglar es relativamente alta y aunque no se tomaron datos del porcentaje de salinidad, Kauffman y colaboradores (2016) mencionan que El Cometa tiene un porcentaje de salinidad de $4 \pm 0.6 \mathrm{ppt}$ en zonas donde se llevó a cabo este estudio, lo que indica que las epífitas de este lugar están soportando relativamente bajos niveles de salinidad, si se compara con los 25-40 ppt que pueden llegar a tener los manglares de las lagunas Celestún, Chelem y Términos de la península de Yucatán (Chuang et al., 2017).
El género con mayor número de especies fue $T i$ llandsia, lo que concuerda con lo encontrado por otros estudios en manglares de México y Guatemala (Gómez y Winkler, 1991; Dejean et al., 1995; Cach-Pérez et al., 2013; Chilpa-Galván et al., 2013). Tillandsia tiene semillas plumosas que le permiten dispersarse fácilmente por el viento, promoviendo su dispersión y debido a que algunas características de los forófitos (altura, DAP, tipo de corteza) están relacionadas con el anclaje de semillas dispersas por el viento, hacen que este género esté mejor adaptado a las condiciones ambientales presentes en los manglares (p. ej. Dejean et al., 1995; García-Franco y Rico-Gray, 1988). Además, la mayor parte de las especies de Tillandsia encontradas son tolerantes a la sequía, debido a su metabolismo del ácido crasuláceo (CAM) (Dejean et al., 1995; Graham y Andrade, 2004; Reyes-García et al., 2012; Chilpa-Galván et al., 2013). Esto a diferencia de otros grupos epífitos, como las orquídeas, cuya germinación y crecimiento dependen de la existencia de los hongos de mycorrhiza en las raíces (Lüttge, 1989). Sin embargo, el rocío por las mañanas podría estar favoreciendo una mayor diversidad de orquídeas epífitas (Jiménez-López, datos sin publicar), ya que esta humedad puede ser absorbida por el velamen de las raíces o los estomas en las hojas (de la Rosa-Manzano et al., 2014). 
Se observó que las epífitas del Cometa son escasas comparadas con selvas bajas de Veracruz, donde se registraron 58 y 69 especies en dos parcelas de 0.15 ha (HietzSeifert et al., 1996) y en bosques mesófilo de montaña con 88-93 especies en tres parcelas de 0.32 ha (Krömer et al., 2014). Sin embargo, son más diversas comparándolas con otros manglares; Cach-Pérez et al. (2013) reportan dos especies en cinco parcelas de 0.1 ha en Yucatán, mientras que Chilpa-Galván et al. (2013) encontraron ocho especies en 11 parcelas de 0.1 ha en selvas bajas en Yucatán. Esto debido a que los bosques mesófilos de montaña tienen una alta heterogeneidad del hábitat, son topográficamente muy accidentadas y cuentan con un dosel cerrado. Estas variables crean microclimas distintos desde la parte alta del dosel hasta el suelo (Krömer et al., 2007a), favoreciendo una amplia distribución vertical en epífitas vasculares. En conjunto estos componentes con otros de tipo ecológico y de especiación, así como condiciones favorables de temperatura, precipitación y humedad favorecen la alta riqueza de epífitas vasculares dentro de este tipo de bosques (Gentry y Dodson, 1987; Benzing, 1990; Kreft et al., 2004; Küper et al., 2004; Zotz, 2005; Krömer et al., 2013).

\section{Estructura y distribución vertical}

Las raíces aéreas y el dosel interior tuvieron la mayor riqueza de especies. Esto puede estar determinado por los mecanismos de dispersión de las epífitas del dosel, cuyas semillas y/o plántulas pueden ser removidas por el viento o la lluvia y al caer alcanzan las raíces, que tienen un área amplia inmediatamente por debajo del forófito, lo cual también estaría explicando la alta similitud florística entre ambas zonas (Gómez y Winkler, 1991; Murren y Ellison, 1996). Este resultado está confirmando parcialmente la segunda hipótesis planteada, ya que en términos de similitud florística, las otras zonas del dosel (IV y V) no están directamente relacionadas con el área de las raíces. Nuestro estudio demuestra por primera vez que la superficie de las raíces aéreas proporciona un nicho adicional para la colonización de epífitas. Esto tiene que ver con mayor disposición del área, puesto que las especies epífitas aumentan con un mayor tamaño de los forófitos (Zotz et al., 2001; Flores-Palacios y García-Franco, 2006; Zotz y Schultz, 2008). Una mayor área provee nichos adicionales y microclimas más favorables para la colonización y crecimiento de epífitas (Krömer et al., 2007a; Cach-Pérez et al., 2013; Taylor y Burns, 2015). Lo anterior concuerda con los resultados de Zimmerman y Olmsted (1992), en árboles con ramas de mayor tamaño tuvieron una mayor colonización de especies epífitas en bosques sucesionalmente inundados, en 8 transectos de $150 \mathrm{~m}^{2}$ en Quintana Roo, México. Aunque la cercanía con la hipersalinidad del agua es un factor que debería limitar esta presencia de epífitas, presumimos que la gran cantidad de lluvia contribuye a eliminar el exceso de sal contenida en las especies epífitas, similar a lo que pasa en manglares de Guatemala (Gómez y Winkler, 1991). Además, las especies epífitas en manglares prefieren las partes bajas de los forófitos, donde se observan microambientes menos variables, con menor incidencia de luz (Reyes-García et al., 2012; CachPérez et al., 2013), a diferencia de los bosques mesófilos de montaña, que al contener un dosel cerrado y mayor humedad proveniente de la niebla, favorecen la presencia de epífitas en partes altas del dosel (Krömer et al., 2007a).

La zona III (dosel interno) es la segunda más rica, la cual puede estar favorecida por una mayor acumulación de material orgánico en las bifurcaciones de las ramas principales (Acebey y Krömer, 2001; Zotz y Andrade, 2002; Krömer et al., 2007a). Esto también contribuye al establecimiento de A. bracteata (Dejean et al., 1995, Dejean y Olmsted, 1997), una bromelia de tipo tanque que fue muy frecuente en el dosel interno de los forófitos, al preferir cortezas bien desarrolladas, bosques maduros con alta riqueza de árboles como lo son en El Cometa y las partes bajas de estos, donde las ramas son más estables para soportar su peso (Schultz, 2005; Goode y Allen, 2009). Por otro lado, la baja riqueza observada en la zona $\mathrm{V}$ puede ser resultado de la ausencia de material orgánico sobre las ramas delgadas, o bien, por el grado alto de luminosidad, periodos prolongados de sequía y altas temperaturas (Acebey y Krömer, 2001; Flores-Palacios y García-Franco, 2008; Hietz, 2010; Reyes-García et al., 2012). 


\section{Relación entre el tamaño del forófito y la rique- za de epífitas}

Las raíces aéreas de $R$. mangle varían en longitud y grosor (Tomlinson, 2016), constituyendo así un espacio disponible más para que sea colonizado por epífitas, ampliando de esta forma sus nichos. Esta situación no está presente en otros tipos de vegetación y es reflejo de la particularidad del sistema de manglar. Aunque en el presente estudio la altura fue la relación más débil, se sabe que el tamaño del árbol hospedero influye positivamente en la riqueza de epífitas, ya que en forófitos con diámetros mayores, el área superficial es más extensa para el establecimiento de las mismas (Flores-Palacios y García-Franco, 2006; Krömer et al., 2007b). Por el contrario, Cach-Pérez et al. (2013) no encuentran una relación entre la altura y la abundancia de bromelias en manglares de Yucatán; no obstante, esto puede ser debido a la estatura baja de los individuos de $R$. mangle, que alcanzan una altura de $1.5 \mathrm{~m}$, mientras que los forófitos de los manglares de la laguna El Cometa presentan una media de $17 \mathrm{~m}$, aumentando el área disponible para la colonización de epífitas. Los forófitos de gran tamaño suelen ser más longevos, y por lo tanto, pueden estar disponibles por un lapso de tiempo mayor a la colonización de estas mismas (Acebey y Krömer, 2001; Flores-Palacios y García-Franco, 2006; Krömer et al., 2007b; Goode y Allen, 2009).

\section{CONCLUSIONES}

El manglar de la laguna El Cometa contiene una riqueza relativamente alta de epífitas. Las familias mejor representadas son Bromeliaceae y Orchidaceae, las cuales son generalmente las más abundantes en este tipo de ecosistema en México. El tamaño de los forófitos determinó el establecimiento de epífitas, ya que los arboles de tallas mayores son colonizados por más de estas especies. Se demuestra la importancia de las raíces aéreas de $R$. mangle como un nicho adicional para la colonización de epífitas y no solo como soporte al suelo inestable de este ecosistema. No obstante, existen otras variables no consideradas en este estudio (p. ej., precipitación, humedad y salinidad, entre otros) que pueden influir en la riqueza de epífitas dentro del manglar. Se recomienda ampliar los estudios ecológicos de estas mismas en estos ecosistemas de México y el Neotrópico e incluir a las raíces aéreas y factores ambientales como unas variables importantes que potencialmente determinan la riqueza de especies.

\section{CONTRIBUCIONES DE AUTORES}

DAJ, RRV y JGC idearon y diseñaron el estudio. NMM y GO interpretaron los datos, DAJ y GO realizaron los análisis. DAJ escribió el manuscrito y todos los autores contribuyeron a la revisión y aprobación del manuscrito final.

\section{FINANCIAMIENTO}

Este estudio fue apoyado por el Centro del Cambio Global y la Sustentabilidad del Sureste (CCGSS), proyecto retos para la sustentabilidad en la cuenca del rio Usumacinta, ecosistemas, cambio climático y respuesta social (FOMIX-TAB-2012-C28-194316).

\section{AgRADECIMIENTOS}

A Rubén Martínez Camilo y dos revisores anónimos por los comentarios que enriquecieron este manuscrito. A Jonathan V. Solorzano por realizar el mapa de la zona de estudio. Ana Fernández, Gerardo Cervantes, Jorge Navarro, Marco A. Domínguez y don Bruno Chávez por la asistencia en campo. A Ivon Ramírez Murillo por el apoyo en identificación en Bromeliaceae.

\section{Literatura Citada}

Acebey, A. y T. Krömer. 2001. Diversidad y distribución vertical de epífitas en los alrededores del campamento río Eslabón y de la laguna Chalalán, Parque Nacional Madidi, Dpto. La Paz, Bolivia. Revista de la Sociedad Boliviana de Botánica 3: 104-123.

Benzing, D. H. 1990. Vascular epiphytes: general biology and related biota. Cambridge University Press. Cambridge, UK. 346 pp.

Cach-Pérez, M. J., J. L. Andrade, N. Chilpa-Galván, M. TamayoChim, R. Orellana y C. Reyes-García. 2013. Climatic and structural factors influencing epiphytic bromeliad 
community assemblage along a gradient of water-limited environments in the Yucatán Peninsula, Mexico. Tropical Conservation Science 6(2): 283-302.

Chilpa-Galván, N., M. Tamayo-Chim, J. L. Andrade y C. Reyes-García. 2013. Water table depth may influence the asymmetric arrangement of epiphytic bromeliads in a tropical dry forest. Plant Ecology 214(8): 1037-1048. DOI: http://dx.doi.org/10.1007/s11258-013-0229-3

Chuang, P. C., M. B. Young, A. W. Dale, L. G. Miller, J. A. Herrera Silveira y A. Paytan. 2017. Methane fluxes from tropical coastal lagoons surrounded by mangroves, Yucatán, Mexico. Journal of Geophysical Research: Biogeosciences 122: DOI: http://dx.doi. org/10.1002/2017JG003761.

de la Rosa-Manzano, E., J. L. Andrade, G. Zotz y C. ReyesGarcía. 2014. Epiphytic orchids in tropical dry forests of Yucatán, Mexico-Species occurrence, abundance and correlations with host tree characteristics and environmental conditions. Flora-Morphology, Distribution, Functional Ecology of Plants 209(2): 100-109. DOI: http://dx.doi. org/10.1016/j.flora.2013.12.002

Dejean, A. y I. Olmsted. 1997. Ecological studies on Aechmea bracteata (Swartz) (Bromeliaceae). Journal of Natural History 31(9): 1313-1334. DOI: http://dx.doi. org/10.1080/00222939700770741

Dejean, A., I. Olmsted y R. R. Snelling. 1995. Tree-EpiphyteAnt relationships in the low inundated forest of Sian Ka'an Biosphere Reserve, Quintana Roo, Mexico. Biotropica 27(1): 57-70. DOI: http://dx.doi.org/10.2307/2388903

Flores-Palacios, A. y J. G. García-Franco. 2006. The relationship between tree size and epiphyte richness: testing four different hypotheses. Journal of Biogeography 33(2): 323-330. DOI: http://dx.doi.org/10.1111/j.13652699.2005.01382.x

Flores-Palacios, A. y J. G. García-Franco. 2008. Habitat isolation changes the beta diversity of the vascular epiphyte community in lower montane forest, Veracruz, Mexico. Biodiversity and Conservation 17(1): 191-207. DOI: http://dx.doi.org/10.1007/s10531-007-9239-6

Garcia-Franco, J. G. y V. Rico-Gray. 1988. Experiments on seed dispersal and deposition patterns of epiphytes. The case of
Tillandsia deppeana Steudel (Bromeliaceae). Phytologia Plainfield NJ 65(1): 73-78.

Gentry, A. H. y C. H. Dodson. 1987. Diversity and biogeography of neotropical vascular epiphytes. Annals of the Missouri Botanical Garden 74(2): 205-233. DOI: http://dx.doi. org/10.2307/2399395

Giesen, W., S. Wulffraat, M. Zieren y L. Scholten. 2007. Mangrove guidebook for Southeast Asia. Mangrove guidebook for Southeast Asia. Bangkok, Thailandia. 769 pp.

Giri, C., E. Ochieng, L. L. Tieszen, Z. Zhu, A. Singh, T. Loveland, J. Masek y N. Duke. 2011. Status and distribution of mangrove forests of the world using earth observation satellite data. Global Ecology and Biogeography 20: 154-159. DOI: http://dx.doi.org/10.1111/j.14668238.2010.00584.x

Gómez, M. A. y S. Winkler. 1991. Bromelias en manglares del Pacífico de Guatemala. Revista de Biología Tropical 39(2): 207-214.

Goode, L. K. y M. F. Allen. 2009. Seed germination conditions and implications for establishment of an epiphyte, Aechmea bracteata (Bromeliaceae). Plant Ecology 204(2): 179-188. DOI: http://dx.doi.org/10.1007/s11258009-9582-7

Gradstein, S. R., N. M. Nadkarni, T. Krömer, I. Holz y N. Nöske. 2003. A protocol for rapid and representative sampling of vascular and non-vascular epiphyte diversity of tropical rain forest. Selbyana 24(1): 105-111.

Graham, E. A. y J. L. Andrade. 2004. Drought tolerance associated with vertical stratification of two co-occurring epiphytic bromeliads in a tropical dry forest. American Journal of Botany 91(5): 699-706. DOI: http://dx.doi. org/10.3732/ajb.91.5.699

Guadarrama-Olivera, M. A. y G. Ortiz-Gil. 2000. Flora de la reserva de la biósfera de los "Pantanos de Centla", en el estado de Tabasco, México. Informe final SNIBCONABIO. Proyecto No. L138. Universidad Juárez Autónoma de Tabasco. Tabasco, México. http:// www.inecc.gob.mx/descargas/publicaciones/179.pdf. (consultado abril 2015)

Haro-Carrión, X., T. Lozada, H. Navarrete, G. H. J. de Koning. 
2009. Conservation of vascular epiphyte diversity in shade Cacao Plantations in the Chocó Region of Ecuador. Biotropica 41(4): 520-529. DOI: http://dx.doi. org/10.1111/j.1744-7429.2009.00510.x

Hietz, P. 2010. Ecology and ecophysiology of epiphytes in tropical montane cloud forests. In: Bruijnzeel, L. A., F. N. Scatena y L. S. Hamilton (eds.). Tropical Montane Cloud Forests: Science for Conservation and Management. Cambridge University Press. Nueva York, USA. pp.6776.

Hietz-Seifert, U., P. Hietz y S. Guevara. 1996. Epiphyte vegetation and diversity on remnant trees after forest clearance in southern Veracruz, Mexico. Biological Conservation 75(2): 103-111. DOI: http://dx.doi. org/10.1016/0006-3207(95)00071-2

INE. 2000. Programa de manejo de la Reserva de la Biosfera "Pantanos de Centla". Instituto Nacional de Ecología. México, D.F., México. 222 pp.

Johansson, D. 1974. Ecology of vascular epiphytes in West African Rain Forest. Acta Phytogeographica Suecica 59: $1-129$.

Kauffman, J. B., H. H. Trejo, M. D. C. J. Garcia, C. Heider y W. M. Contreras. 2016. Carbon stocks of mangroves and losses arising from their conversion to cattle pastures in the Pantanos de Centla, Mexico. Wetlands Ecology and Management 24(2): 203-216. DOI: http://dx.doi. org/10.1007/s11273-015-9453-z

Kreft, H., N. Köster, W. Küper, J. Nieder y W. Barthlott. 2004. Diversity and biogeography of vascular epiphytes in Western Amazonia, Yasuní, Ecuador. Journal of Biogeography 31(9): 1463-1476. DOI: http://dx.doi. org/10.1111/j.1365-2699.2004.01083.x

Krömer, T., A. Acebey, J. Kluge y M. Kessler. 2013. Effects of altitude and climate in determining elevational plant species richness patterns: a case study from Los Tuxtlas, Mexico. Flora-Morphology, Distribution, Functional Ecology of Plants 208(3): 197-210. DOI: http://dx.doi. org/10.1016/j.flora.2013.03.003

Krömer, T., J. G. García-Franco y T. Toledo-Aceves. 2014. Epífitas vasculares como bioindicadores de la calidad forestal: impacto antrópico sobre su diversidad y composición. In: González-Zuarth, C. A., A. Vallarino, J. C. Pérez-Jimenez y A. M. Low-Pfeng (eds.). Bioindicadores: guardianes de nuestro futuro ambiental. Instituto Nacional de Ecología y Cambio Climático (INECC) - El Colegio de la Frontera Sur (ECOSUR). México, D.F. y Campeche, México. pp. 606-623

Krömer, T., M. Kessler y S. R. Gradstein. 2007a. Vertical stratification of vascular epiphytes in submontane and montane forest of the Bolivian Andes: the importance of the understory. Plant Ecology 189(2): 261-278. DOI: http://dx.doi.org/10.1007/s11258-006-9182-8

Krömer, T., S. R. Gradstein y A. Acebey. 2007b. Diversidad y ecología de epífitas vasculares en bosques montanos primarios y secundarios de Bolivia. Ecología en Bolivia 42(1): 23-33.

Küper, W., H. Kreft, J. Nieder, N. Köster y W. Barthlott. 2004. Large-scale diversity patterns of vascular epiphytes in Neotropical montane rain forests. Journal of Biogeography 31(9): 1477-1487. DOI: http://dx.doi.org/10.1111/j.13652699.2004.01093.x

Lot, A. y F. Chiang. 1986 Manual de herbario: Administración y manejo de colecciones, técnicas y preparación de ejemplares botánicos. Consejo nacional de flora de México, A.C. México, D.F., México. 342 pp.

Lüttge, U. 1989. Vascular plants as epiphytes. Evolution and ecophysiology. Ecological Studies. Berlin, Germany. 263 pp.

Margalef, R. 1995. Ecología. Omega. Barcelona, España. 945 pp.

Martínez-Meléndez, N., M. A. Pérez-Farrera y A. FloresPalacios. 2008. Estratificación vertical y preferencia de hospedero de las epífitas vasculares de un bosque nublado de Chiapas, México. Revista de Biología Tropical 56(4): 2069-2086.

Miranda, F. y E. Hernández-X. 1963. Los tipos de vegetación de México y su clasificación. Instituto de Biología. México, D.F., México. 176 pp.

Moreno, C. E. 2001. Métodos para medir la biodiversidad. M\&T-Manuales y Tesis SEA. Zaragoza, España. 83 pp.

Murren, C. J. y A. M. Ellison. 1996. Effects of habitat, plant size, and floral display on male and female reproductive success 
of the Neotropical orchid Brassavola nodosa. Biotropica 28(1): 30-41. DOI: http://dx.doi.org/10.2307/2388769

Poungparn, S. yA. Komiyama. 2013. Net ecosystem productivity studies in mangrove forests. Reviews in Agricultural Science 1: 61-64. DOI: http://dx.doi.org/10.7831/ras.1.61

R Core Team. 2015. R: A language and environment for statistical computing, version3.2.3. programa distribuido por el autor. R Foundation for Statistical Computing. Vienna, Austria. https://www.r-project.org/.

Reyes-García, C., M. Mejía-Chang y H. Griffiths. 2012. High but not dry: diverse epiphytic bromeliad adaptations to exposure within a seasonally dry tropical forest community. New Phytologist 193: 745-754. DOI: http:// dx.doi.org/10.1111/j.1469-8137.2011.03946.x

Rodríguez-Rodríguez, E. 2002. Las lagunas continentales de Tabasco. Universidad Juárez Autónoma de Tabasco. Tabasco, México. 245 pp.

Rodríguez-Zúñiga, M. T., C. Troche-Souza, A. D. VázquezLule, J. D. Márquez-Mendoza, B. Vázquez-Balderas, L. Valderrama-Landeros, S. Velázquez-Salazar, M. I. CruzLópez, R. Ressl, A. Uribe-Martínez, S. Cerdeira-Estrada, J. AcostaVelázquez, J. Díaz-Gallegos, R. JiménezRosenberg, L. Fueyo-Mac Donald y C. Galindo-Leal. 2013. Manglares de México/Extensión, distribución y monitoreo. Comisión Nacional para el Conocimiento y Uso de la Biodiversidad. México, D.F., México. 128 pp.

Sanford, W. W. 1968. Distribution of epiphytic orchids in semi-deciduous tropical forest in southern Nigeria. Journal of Ecology 56(3): 697-705. DOI: http://dx.doi. org/10.2307/2258101

Schultz, G. P. 2005. Vascular flora of the El Edén Ecological Reserve, Quintana Roo, Mexico. The Journal of the Torrey Botanical Society 132(2): 311-322. DOI: http:// dx.doi.org/10.3159/1095-5674(2005)132[311:VFOTEE] 2.0.CO;2

SEMARNAT. 2010. NORMA Oficial Mexicana NOM-059-SEMARNAT-2010. Protección ambiental-Especies nativas de México de flora y fauna silvestres-Categorias de riesgo y especificaciones para su inclusión, exclusión o cambioLista de especies en riesgo. Secretaría del Medio Ambiente y Recursos Naturales. Diario Oficial de la Federación.
Cd. Mx., México. http://dof.gob.mx/nota_detalle.php?co digo $=5173091 \&$ fecha $=30 / 12 / 2010$

Spalding, M., M. Kainuma y L. Collins. 2010. World atlas of mangroves. Earthscan. London, UK and Washington, DC., USA. 305 pp.

Taylor, A. y K. Burns. 2015. Epiphyte community development throughout tree ontogeny: an island ontogeny framework. Journal of Vegetation Science 26(5): 902-910. DOI: http:// dx.doi.org/10.1111/jvs.12289

Tomlinson, P. B. 2016. The botany of mangroves. 2 ed. Cambridge University Press. Cambridge, UK. 413 pp.

Valdez-Hernández, J. I. 2000. Flora vascular de los manglares de Marismas Nacionales, Estado de Nayarit. Comisión Nacional para el Conocimiento y Uso de la Biodiversidad. México, D.F., México. http:// www.conabio.gob.mx/institucion/proyectos/resultados/InfS131.pdf (consultado marzo de 2015).

Wolf, J. H. D., S. R. Gradstein y N. M. Nadkarni. 2009. A protocol for sampling of vascular epiphytes richness and abundance. Journal of Tropical Ecology 25(2): 107-121. DOI: http://dx.doi.org/10.1017/S0266467408005786

Zimmerman, J. K. y I. C. Olmsted. 1992. Host tree utilization by vascular epiphytes in a seasonally inundated forest (Tintal) in Mexico. Biotropica 24(3): 402-407. DOI: http://dx.doi.org/10.2307/2388610

Zotz, G. 2005. Vascular epiphytes in the temperate zones - a review. Plant Ecology 176(2): 173-183. DOI: http:// dx.doi.org/10.1007/s11258-004-0066-5

Zotz, G. 2013. The systematic distribution of vascular epiphytes - a critical update. Botanical Journal of the Linnean Society 171(3): 453-481. DOI: http://dx.doi.org/10.1111/ boj. 12010

Zotz, G. 2016. Physiological ecology. In: Zotz, G. (ed.). Plants on plants - The biology of vascular epiphytes. Springer. Cham, Switzerland. Pp. 95-137. DOI: http://dx.doi. org/10.1007/978-3-319-39237-0

Zotz, G. y J. L. Andrade. 2002. La ecología y la fisiología de las epifitas y las hemiepífitas. In: Guariguata, M. R. y G. H. Kattan (eds.). Ecología y conservación de bosques neotropicales. Editorial Libro Universitario Regional. Cartago, Costa Rica. Pp. 271-296. 
Zotz, G. y N. Reuter. 2009. The effect of exposure to sea water on germination and vegetative growth of an epiphytic bromeliad. Journal of Tropical Ecology 25(3): 311-319. DOI: https://doi.org/10.1017/S0266467409006014

Zotz, G. y S. Schultz. 2008. The vascular epiphytes of a lowland forest in Panama species composition and spatial structure. Plant Ecology 195(1): 131-141. DOI: https:// doi.org/10.1007/s11258-007-9310-0
Zotz, G., P. Hietz y G. Schmidt. 2001. Small plants, large plants: the importance of plant size for the physiological ecology of vascular epiphytes. Journal of Experimental Botany 52(363): 2051-2056. DOI: https://doi.org/10.1093/ jexbot/52.363.2051 